\title{
UM MECANISMO DE DECISÃO PARA INFERÊNCIA DE CONTEXTO EM AMBIENTES PERVASIVOS DE TRATAMENTO DE SAÚDE
}

\author{
Alessandro Copetti* \\ acopettidic.uff.br
}

\author{
J. C. B. Leite* \\ juliuseic.uff.br
}

\author{
O. Loques ${ }^{\dagger}$ \\ loquesdic.uff.br \\ ${ }^{*}$ Instituto de Computação — Universidade Federal Fluminense (UFF) \\ Niterói, Rio de Janeiro, Brasil \\ ${ }^{\dagger}$ Instituto de Ciência e Tecnologia — Universidade Federal Fluminense (UFF) \\ Rio das Ostras, Rio de Janeiro, Brasil
}

\begin{abstract}
A decision mechanism to context inference in pervasive healthcare environments

This paper presents a Fuzzy approach to monitor the health of a patient in pervasive computing environments. A decision model considers three classes of variables that represent the context information being collected: environmental, physiological and behavioral. A case study of blood pressure monitoring was developed to identify critical situations based on medical knowledge. The solution maintains the interpretability of a set of defined rules, even after a learning phase that proposes adjustments to them. In this phase, the Fuzzy C-Means clustering was chosen to adjust membership functions, using the cluster centers. A medical team evaluated data from 24-hour monitoring of 30 patients and the rating was compared with the results of the system. The proposed approach proved to be individualized, identifying critical events in patients with different levels of blood pressure with an accuracy of $90 \%$ and low rate of false negatives.
\end{abstract}

KEYWORDS: context inference, fuzzy logic, pervasive healthcare

Artigo submetido em 17/08/2010 (Id.: 01170)

Revisado em 12/10/2010, 21/01/2011, 08/02/2011

Aceito sob recomendação do Editor Associado Prof. Ivan Nunes Da Silva

\section{RESUMO}

Este trabalho apresenta uma abordagem utilizando sistemas Fuzzy para o monitoramento de saúde de um paciente em ambientes de computação pervasiva. Um modelo de decisão considera três classes de variáveis que constituem as informações de contexto sendo coletadas: ambientais, fisiológicas e comportamentais. Um estudo de caso de monitoramento da pressão arterial foi desenvolvido para identificar situações críticas com base em conhecimento médico. A solução mantém a interpretabilidade de um conjunto de regras definidas, mesmo após uma fase de aprendizado que propõe ajustes nessas regras. Nessa fase, a técnica de agrupamento Fuzzy C-Means foi escolhida para o ajuste das funções de pertinência, usando os centros dos agrupamentos. Uma equipe médica avaliou dados de monitoramento de 24 horas de 30 pacientes e esta avaliação foi comparada com os resultados do sistema. A abordagem proposta demonstrou ser individualizada, identificando situações críticas em pacientes com diferentes níveis de pressão arterial, com uma acurácia de $90 \%$ e baixa taxa de falsos negativos.

PALAVRAS-CHAVE: inferência de contexto, lógica fuzzy, tratamento de saúde e computação pervasiva 


\section{INTRODUÇÃO}

O aumento da proporção de idosos representa um grande desafio para o sistema de saúde. Atualmente, cerca de $11 \%$ da população mundial é composta por idosos; em 2050, os idosos deverão representar um quinto da população (ONU, 2009). Um problema associado são as doenças crônicas, que no Brasil já atingem 3 em cada 4 idosos (IBGE, 2009). Os pacientes que convivem com doenças crônicas, como, por exemplo, diabetes e doenças cardiovasculares, necessitam de um monitoramento de longa duração em sua vida diária, com grande nível de detalhamento. Como muitos desses idosos moram sozinhos, a residência torna-se o principal local para o monitoramento.

Principalmente com o avanço das tecnologias de sensores sem fio, posicionados tanto nos ambientes como no corpo das pessoas (computação vestível), hoje já é possível projetar novas aplicações de monitoramento remoto. Estas aplicações podem executar em ambientes pervasivos dotados de dispositivos capazes de realizar computação e comunicação. A partir disso, é possível imaginar um ambiente doméstico com unidades de processamento que realizam desde a coleta de dados até a identificação e notificação de emergências. Por sua vez, os dados assim gerados caracterizam determinadas situações de interesse envolvendo objetos, pessoas e lugares, formando o conceito de contexto (Dey, 2001). A correta transformação desses dados em informações de alto nível é um dos principais requisitos para um monitoramento eficiente.

Para um melhor entendimento, exemplifica-se neste artigo duas situações proporcionadas pelo uso da computação pervasiva no ambiente domiciliar e que remetem a desafios relacionados à inferência de contexto. Na primeira situação, um idoso é monitorado de forma não intrusiva por sensores de sinais vitais acoplados a seu corpo, os quais coletam dados da pressão arterial e da frequência cardíaca, entre outras informações. Em determinado momento, ocorre uma alteração dos seus sinais vitais, sendo que, nos minutos prévios, teriam sido detectados uma temperatura ambiente alta e um aumento considerável na atividade física do paciente. Já na outra situação analisada, uma grande variação dos sinais vitais é acompanhada de uma possível queda do paciente. Em ambas situações, caracterizadas como críticas, uma notificação é direcionada a um profissional de saúde, que, por sua vez, procura ter melhor conhecimento da situação, e.g., entrando em contato com o paciente.

Nesses dois exemplos, pode-se identificar três classes de variáveis envolvidas nas informações de contexto: ambientais, fisiológicas e comportamentais. Se após a coleta das variáveis for realizada uma fusão de dados, pode-se caracterizar melhor o estado do paciente, classificando-o, inicialmente, como normal, alerta ou emergência. Dessa forma, há a necessidade de mecanismos de decisão mais consistentes que infiram e caracterizem as situações de monitoramento, evitando alarmes falsos ${ }^{1}$.

Nos trabalhos científicos investigados nesta pesquisa não foram propostas abordagens de monitoramento a distância que realizem a fusão de dados ambientais, fisiológicos e comportamentais para a identificação em tempo real e contínua de situações críticas de um paciente idoso. Tais características não são contempladas por propostas que monitorem o paciente em ambientes pervasivos (Sneha e Varshney, 2009; Lee et al., 2008). Este artigo propõe um mecanismo de decisão para inferência de contexto em aplicações de monitoramento de saúde. O mecanismo proposto agrega conhecimento médico, é individualizado e se ajusta às variações de sinais vitais conforme a atividade que o paciente realiza. O trabalho define um modelo denominado de Decisão, de onde derivam dois módulos principais: um Módulo de Inferência que reconhece situações críticas e um Módulo de Aprendizado que propõe ajustes no mecanismo de inferência.

O trabalho está estruturado da seguinte forma: a segunda Seção faz um estudo de pesquisas na área de monitoramento domiciliar usando computação pervasiva. A terceira Seção apresenta o Modelo de Decisão, de onde se originam o Módulo de Inferência e o Módulo de Aprendizado, apresentados nas Seções 4 e 5, respectivamente. A Seção 6 realiza a avaliação da proposta de monitoramento. Por fim, a Seção 7 apresenta as conclusões.

\section{MONITORAMENTO DOMICILIAR DOS SINAIS VITAIS}

Os sistemas atuais de tratamento de saúde em casa exigem que a pessoa informe emergências através do acionamento de alarmes, como, por exemplo, por meio de um botão de pânico. Ou então, que os sistemas reajam quando limites pré-fixados de sinais vitais são ultrapassados, como em Leijdekkers et al. (2007). Há, nesses casos, a necessidade de identificar situações críticas com maior eficiência, tendo um compromisso entre acionar alarmes e ser tolerante às variações de sinais fisiológicos.

Estas variações podem ser influenciadas pelo comportamento ou pelas condições do ambiente. Assim, por exemplo, enquanto a pessoa está caminhando ou realizando uma atividade doméstica, naturalmente aumenta a sua pressão arterial (da Nobrega, 2005); além disso, a temperatura do ambiente também tem influência sobre a pressão arterial (Mion et al., 2004). Para realizar esse monitoramento ciente do contexto e aprender padrões de comportamento individuais e re-

\footnotetext{
${ }^{1}$ Neste trabalho, os alertas e as emergências são denominados 'alarmes'.
} 
conhecer atividades, os trabalhos de pesquisa têm usado algoritmos de aprendizado (Marschollek et al., 2007; Pentney et al., 2006).

No entanto, pouco avanço foi realizado até hoje na fusão das diferentes classes de variáveis envolvidas no monitoramento. Em Pärkkä et al. (2009) é apresentado um estudo explorando o relacionamento entre variáveis psicológicas e fisiológicas no monitoramento domiciliar; algumas variáveis comportamentais também são consideradas e realizadas correlações entre as variáveis. Por exemplo, níveis altos de estresse estão associados com menor tempo de sono. Já em Duchêne et al. (2007), é construído o perfil comportamental da pessoa monitorada em casa, investigando o relacionamento entre variáveis comportamentais e fisiológicas, em especial, a frequência cardíaca.

No caso de um controle da pressão arterial, duas formas são estabelecidas pela Sociedade Brasileira de Cardiologia, que consideram a pessoa sendo monitorada fora do consultório médico: MRPA (Monitorização Residencial da Pressão Arterial) e MAPA (Monitorização Ambulatorial da Pressão Arterial). Apesar de serem usadas principalmente para esclarecimento diagnóstico (Mion et al., 2004), estas ferramentas podem estabelecer a base de conhecimento para definir um monitoramento de longa duração. A MRPA orienta que o paciente faça três medições pela manhã e três à noite, durante cinco dias. Já a MAPA, é realizado durante 24 horas, podendo atingir em torno de 80 medições, com intervalos de 20 min durante a vigília e 30 min durante o sono (Mion et al., 2004). Os pacientes são orientados em ambos os métodos a anotar sua atividade, enquanto são feitas medições da pressão sistólica e diastólica e frequência cardíaca.

Para monitorar as variáveis envolvidas na MAPA, em um recente trabalho (Sztajnberg et al., 2009), definiu-se uma arquitetura que inclui serviços sensíveis ao contexto, que possibilitam descobrir e gerenciar recursos (sensores e dispositivos). Assim, as aplicações de monitoramento de saúde podem contar com uma infraestrutura para gerenciar os recursos envolvidos. Em outro trabalho (Copetti et al., 2009), estruturouse na forma de um framework as funcionalidades relevantes para um monitoramento em um cenário de assistência domiciliar, bem como, introduziu-se os componentes necessários para tratar as diversas fontes de dados.

\section{UM MODELO DE DECISÃO PARA O MO- NITORAMENTO}

Para que um Modelo de Decisão identifique situações críticas, caracterizadas principalmente por alterações nos sinais vitais, um requisito fundamental é incorporar conhecimento médico. Uma forma simples para uma representação desse conhecimento, que pode ser bem entendida por médicos, são as regras se-então. Assim, elaborou-se regras a partir do conhecimento publicado em diretrizes médicas (SBC, 2010) e da experiência de especialistas em saúde participantes do grupo de pesquisa dos autores desse trabalho. Alguns exemplos são:

- Se a média da pressão sistólica for maior que $135 \mathrm{mmHg}$ e a da diastólica for maior que $85 \mathrm{mmHg}$, então o paciente é considerado hipertenso (SBC, 2005)

- Se está realizando tarefas/atividades domésticas, então a pressão sistólica deve subir no máximo $10,7 \mathrm{mmHg}$ da média e a diastólica $6,7 \mathrm{mmHg}$ (Mion et al., 2004)

- Se está dormindo, então a pressão sistólica deve ficar no máximo $-10 \mathrm{mmHg}$ da média e a diastólica $-7,6 \mathrm{mmHg}$ (SBC, 2005)

- Se a temperatura ambiente está alta e a pressão arterial está baixa, então é considerado um alerta leve

As regras para o Modelo de Decisão podem associar as três classes de variáveis: ambientais, fisiológicas e comportamentais (a atividade da pessoa). Um elemento importante da regra é a possibilidade de usar a média de um determinado sinal vital. Este recurso permite uma generalização da regra aplicável em pacientes com diferentes níveis de dados fisiológicos.

Buscando uma simplificação da inerente complexidade do problema, elaborou-se um sistema de decisão que implementa um Modelo de Decisão, apresentado na Figura 1. As diferentes classes de variáveis são tratadas, inicialmente, por um componente de Pré-processamento, o qual usa informações do perfil do paciente. Essas informações podem ser, por exemplo, as médias de sinais vitais, para calcular os desvios em relação a essas médias. Em seguida, o componente de Inferência realiza a inferência de contexto para determinar o estado do paciente. Após uma determinada quantidade de medições, o componente de Aprendizado pode atuar e uma atualização nas variáveis pode ser proposta ao médico. Essa quantidade de medições só poderá ser determinada pelo médico após um tempo de execução do sistema.

Quanto ao estado do paciente, o estado de normalidade ("normal") significa que a entrada de dados produziu um valor esperado ou razoável para o paciente. Já os "alertas" e as "emergências" são categorizadas como situações críticas, mas em níveis diferentes. Um alerta serve para identificar variações e facilitar a análise médica. As emergências determinam que o sistema solicite, logo em seguida, uma nova medição para confirmar a primeira. Após isso, a emergência pode lançar um aviso a um médico ou a um serviço de pronto atendimento. 


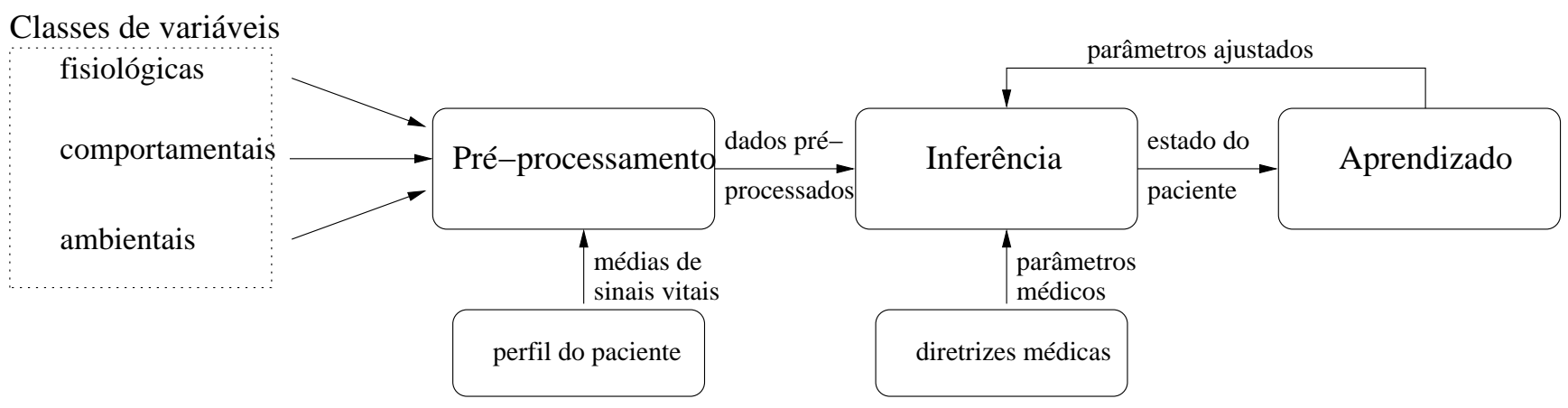

Figura 1: Modelo de Decisão

O projeto dos componentes de Inferência e de Aprendizado é implementado pelos respectivos módulos que são apresentados na Seção 4 e na Seção 5, respectivamente.

\section{MÓDULO DE INFERÊNCIA}

Esta Seção descreve as variáveis, regras e métodos utilizados no Módulo de Inferência. O projeto deste Módulo considera as diretrizes para os exames de MAPA e de MRPA. No entanto, a solução desenvolvida nesse trabalho difere em duas características desses dois tipos de monitoramento. Na proposta aqui concebida, o controle da pressão arterial é de longa duração, e concebeu-se um ambiente de monitoramento com sensores na residência e no corpo da pessoa.

A dinâmica do comportamento humano e a inerente variabilidade dos sinais vitais e as incertezas associadas com os dados coletados dos sensores devem ser consideradas quando definidas as variáveis de interesse. Adicionalmente, o conhecimento médico é expresso de uma maneira que torna a aplicação de conceitos rígidos inapropriada nesse tipo de problema. Considerando esse contexto, adotou-se um modelo em lógica fuzzy para representar as variáveis relevantes e para implementar a função de tomada de decisão do Módulo.

As variáveis presentes na MAPA estão restritas à pressão arterial (PA), à frequência cardíaca (FC) e à atividade que é informada pelo paciente. Assim, as variáveis fisiológicas escolhidas para fazerem parte da proposta aqui apresentada são: pressão arterial sistólica (PAS), pressão arterial diastólica (PAD) e frequência cardíaca. Essas variáveis podem ser facilmente coletadas e mudam rapidamente de acordo com o estado do paciente. Entre os fatores ambientais (luz, temperatura, ruído e umidade, entre outros), escolheu-se a temperatura ambiente, por ter maior influência na saúde do paciente e ser de fácil obtenção. Para a variável atividade do paciente, considerou-se: dormindo, em repouso, comendo, caminhando e em atividade doméstica. Como não se pretende explorar técnicas de reconhecimento de atividade neste tra- balho, assumiu-se que um subsistema fica responsável por informar a atividade para o sistema.

Como parte de um protocolo de monitoramento (a ser definido futuramente em outro trabalho), o exame de MAPA (de 24 horas) é realizado no paciente. O exame serve principalmente para atualizar a média de pressão arterial do paciente. Após isso, o sistema de monitoramento usa essa média de pressão para subtrair de cada nova medição. Com isso, tem-se um desvio da média, que faz parte da composição das regras.

As variáveis fisiológicas foram modeladas associadas às variáveis comportamentais ou ambientais. Um exemplo é quando se usa as recomendações médicas da pressão arterial para uma pessoa que realiza atividade doméstica (Figura 2). Vê-se que, uma vez reconhecida a atividade doméstica sendo realizada pelo paciente nos minutos precedentes à medição, a medida de PAS com desvio um pouco superior à média pode ser considerada normal.

Os conjuntos 'muitoBaixo' e 'muitoAlto' possuem funções de pertinência, respectivamente, zmf ( $z$-shaped membership function) e smf (s-shaped membership function), enquanto os demais conjuntos são trapezoidais. Uma outra alternativa seriam funções trapezoidais para todos os conjuntos. No entanto, explorou-se o uso das formas em ' $z$ ' e 's' para a transição do alerta para emergência. A justificativa é de que essa transição não é gradual, pois há a necessidade de um "suficientemente muitoAlto" para ir para uma emergência. Esse comportamento é esperado pela área médica, pois, na ocorrência de uma emergência, as atitudes tomadas são bem diferentes das que se emprega em um alerta. No entanto, essa escolha deve ser mais bem investigada em um trabalho futuro, pois a diferença em relação a uma trapezoidal é muito pequena.

A variável "atividade" é tratada como um singleton, isto é, somente os valores inteiros que representam cada atividade tem uma pertinência total. Com exceção da atividade de se 


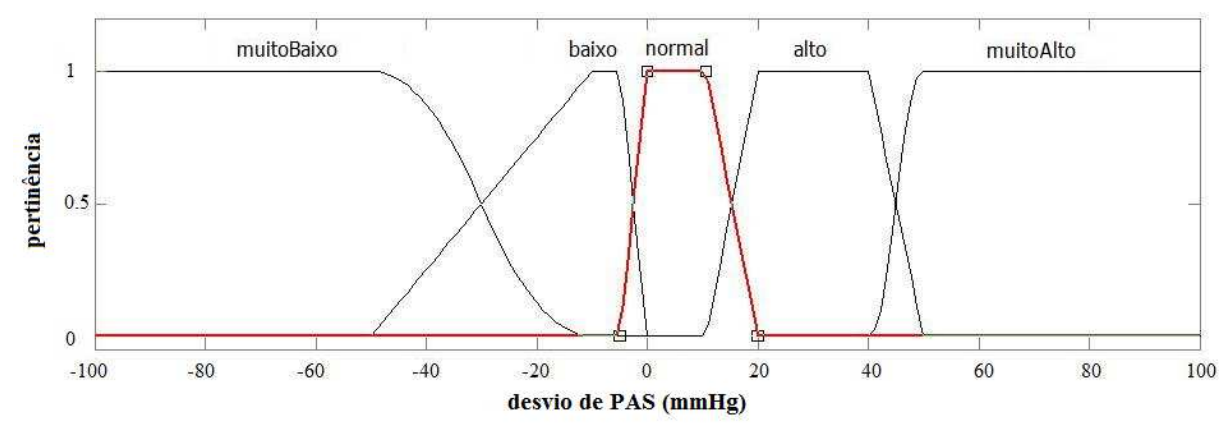

Figura 2: Desvio da Pressão Arterial Sistólica enquanto a pessoa realiza uma atividade doméstica

alimentar, as outras atividades estão relacionadas com o movimento, desde dormir até varrer a casa (atividade doméstica). Isso não exclui, porém, outras possibilidades que venham a ser incorporadas ao sistema, como telefonar ou tomar um medicamento.

A variável tempo é necessária durante a execução do sistema para uma análise mais apurada dos casos de alertas ou de emergências. As emergências têm uma maior significância quando são confirmadas por medições sucessivas em um curto espaço de tempo. Já nos alertas, uma concentração em um determinado período pode ser um fato a ser estudado clinicamente. Um procedimento que faz uma avaliação considerando o tempo pode ser implementado após a inferência da medição. Para esta pesquisa, enfocou-se somente a inferência da medição isolada.

A configuração do sistema de inferência fuzzy foi aperfeiçoada durante o desenvolvimento do trabalho. A configuração final possui os seguintes parâmetros: o sistema de 'inferência' é o 'Mamdani'; na 'implicação' é utilizado o operador 'mínimo'; na 'agregação', o operador 'soma' foi utilizado; o 'bissetor' foi o método de 'defuzificação' empregado. Esses parâmetros foram interessantes para o tipo de problema e direcionados para obter uma saída do sistema gradual em cada estado do paciente. Os parâmetros foram estabelecidos empiricamente levando em consideração as estratégias do Modelo de Decisão.

A definição dos pesos para cada regra também tem papel decisivo na solução. Esses pesos refletem os requisitos do problema quanto à individualização e à identificação de situações críticas. Os critérios adotados para a definição dos pesos são os seguintes: as regras com as médias do paciente devem ter maior peso do que as regras que valem para todos os pacientes (denominadas regras genéricas); as situações de emergência têm prioridade em relação às situações de alerta ou de normalidade; as regras que não incluem a pressão arterial recebem um peso mais baixo, devido à maior importância clínica da pressão arterial.
Um exemplo de regra que não contempla a pressão arterial, mas que relaciona sinais vitais com variáveis ambientais, é: se a frequência cardíaca é alta e a temperatura ambiente é alta, então a ação/estado do paciente é normal (o peso atribuído a essa regra é 0,1$)$.

Um total de 63 regras fazem parte da solução. Essa grande quantidade se justifica pela repetição das regras considerando cada uma das 5 atividades. Para cada atividade tem-se 10 regras que tratam a PAS e a PAD. As 13 regras restantes incluem: regras que reforçam as situações de emergência (denominadas de regras de reforço), mas específicas para os pacientes hipertensos; regras genéricas; e regras com variáveis que não envolvam a pressão arterial. Todas as 63 regras fazem parte da base de regras linguísticas e são avaliadas para inferir o estado do paciente.

\section{MÓDULO DE APRENDIZADO}

O objetivo deste Módulo é personalizar os parâmetros das regras para evitar o lançamento de alarmes desnecessários, usando, para isso, a experiência do médico e os dados de um longo tempo de monitoramento. Ao mesmo tempo, o sistema deve preservar regras de emergência para assegurar confiabilidade na identificação dos casos relevantes que demandem uma notificação, isto é, evitar casos de falsos negativos.

O sistema deve encontrar novos valores toleráveis, mas que não considerem valores extremos já ocorridos. Estas alterações devem ser incrementais, tendo prudência na proposta de ajuste.

Um ajuste não deve gerar conflitos entre regras, portanto o aprendizado deve ser limitado e, ainda, supervisionado por um especialista. Com isso, a necessidade de clareza nas regras (no sentido de transparência ou interpretabilidade das regras) deve ser cumprida. Ajustes que alterem a formação inicial das regras não devem ser propostos, respeitando também os termos adjacentes das variáveis. 
Caso seja adotado uma abordagem de adaptação das regras fuzzy, tem-se dois principais exemplos de técnicas: Neurofuzzy ou Fuzzy Genéticos (Hoffmann, 2001). Trabalhos recentes têm explorado a Neuro-fuzzy, usando particionamento do espaço de entrada (Figueiredo et al., 2007), e inclusive em ambientes sensíveis ao contexto (Anagnostopoulos e Hadjiefthymiades, 2010). Apesar das técnicas de Neuro-fuzzy e fuzzy genéticos obterem bons resultados em várias áreas em que são aplicadas, elas podem produzir mudanças significativas no conjunto de regras iniciais de um sistema de monitoramento de saúde. No contexto do trabalho, essas mudanças têm que ser minimizadas para preservar a interpretabilidade das regras, buscando uma solução que não crie novas regras e use poucas variáveis no antecedente da regra.

\subsection{Geração de dados sintéticos para o monitoramento de sinais vitais}

Para construir um protótipo para o módulo de Aprendizado é importante executar testes. Isso somente é possível com dados sintéticos. O principal objetivo da preparação é obter uma quantidade suficiente de dados realísticos para que os resultados do módulo sejam estatisticamente válidos. Uma alternativa, então, é aumentar essa quantidade por geração de dados sintéticos.

Para tanto, é fundamental ser gerado dados sintéticos que se aproximem dos dados reais. Um passo muito importante nessa direção é a geração com base nos dados de MAPA, que é uma monitorização bem estabelecida na prática médica.

O problema torna-se ainda mais complexo no controle da pressão arterial. Assim, é de grande importância responder a duas questões: a) Os dados sintéticos de PAS ou de PAD possuem a mesma distribuição de probabilidade dos dados originais? b) Os dados sintéticos mantêm a correlação entre PAS e PAD verificada nos dados originais?

Ao ser analisada a distribuição dos dados de MAPA foi identificada uma distribuição normal, mas isso só é válido quando os dados são restritos a somente uma atividade específica. Essa constatação é razoável, pois sabe-se que, na maior parte das pessoas, os valores mais altos de pressão são obtidos na vigília, e os mais baixos, no sono, da mesma forma que o esforço físico pode elevar os sinais vitais. Cabe ressaltar que a atividade que consta na MAPA depende da informação dada pelo paciente e, muitas vezes, essa informação não é dada ou é dada de forma incorreta. Mesmo assim, nos dados que se têm disponíveis, a distribuição normal é encontrada somente quando se separa os dados por atividade.

Então, o emprego da distribuição normal na geração de dados sintéticos aumenta a possibilidade de obter dados mais próximos dos dados reais e permite serem testados no meca- nismo de aprendizagem. Outro argumento é de que a distribuição normal já foi experimentada em outros trabalhos de análise de sinais fisiológicos (El-Samahy et al., 2006; Norris, 2006; Tsipouras et al., 2007).

Quanto a correlação entre os sinais vitais, sabe-se do grande desafio que isso representa. A correlação depende de paciente para paciente: quando um sinal vital aumenta, em algumas pessoas, outro sinal vital também aumenta; dependendo de outros fatores, um sinal vital pode subir e outro pode descer. Essa correlação deve ser preservada nos dados sintéticos, a fim de reproduzir, o mais próximo da realidade, os sinais vitais do paciente.

$\mathrm{Na}$ fase de implementação da geração de dados sintéticos com as características dos dados de MAPA, usou-se o programa de simulação GenData (Ruscio e Haslam, 2006), que gera dados preservando a correlação do conjunto de dados. Por fim, um processo de eliminação/filtragem nos dados sintéticos é realizado, mais especificamente nos valores de pressão arterial, retirando valores praticamente impossíveis de acontecer para cada paciente, considerando uma margem de segurança.

\subsection{Descrição do Módulo de Aprendizado}

Ainda com relação à individualização de cada paciente, é necessário conceber um processo de aprendizado que pode atualizar o Módulo de Inferência com o objetivo de considerar casos em que se denominou de alertas leves. Alertas leves são alertas fracos gerados pelo sistema que, em certas condições, podem ser considerados como normais. Um exemplo prático de necessidade de ajuste é o paciente ter usualmente um descenso noturno muito elevado, o que deveria ser ajustado no sistema para não gerar alarmes desnecessários. Situação semelhante ocorre com pacientes que sofrem com picos moderados de pressão, que, por avaliação médica, não são considerados graves.

A Figura 3 apresenta o fluxo do mecanismo de decisão para inferência de contexto. O processo de aprendizado ocorre sob supervisão médica. Essa intervenção é facilitada pelo parâmetro que define o conceito para alertas leves. A necessidade de usar uma quantidade substancial de dados para aprender é solucionada por considerar os dados de MAPA e os dados sintéticos. Ao final, o médico confirma as alterações nas regras propostas pelo sistema. Da mesma forma, uma mudança para um conjunto de regras mais rígido ou para um conjunto mais tolerante fica a cargo do médico. O mecanismo como um todo evidencia um dos pontos mais importantes do sistema: o aprendizado é individualizado e, ao longo do tempo, personaliza a tomada de decisão sobre situações críticas. 


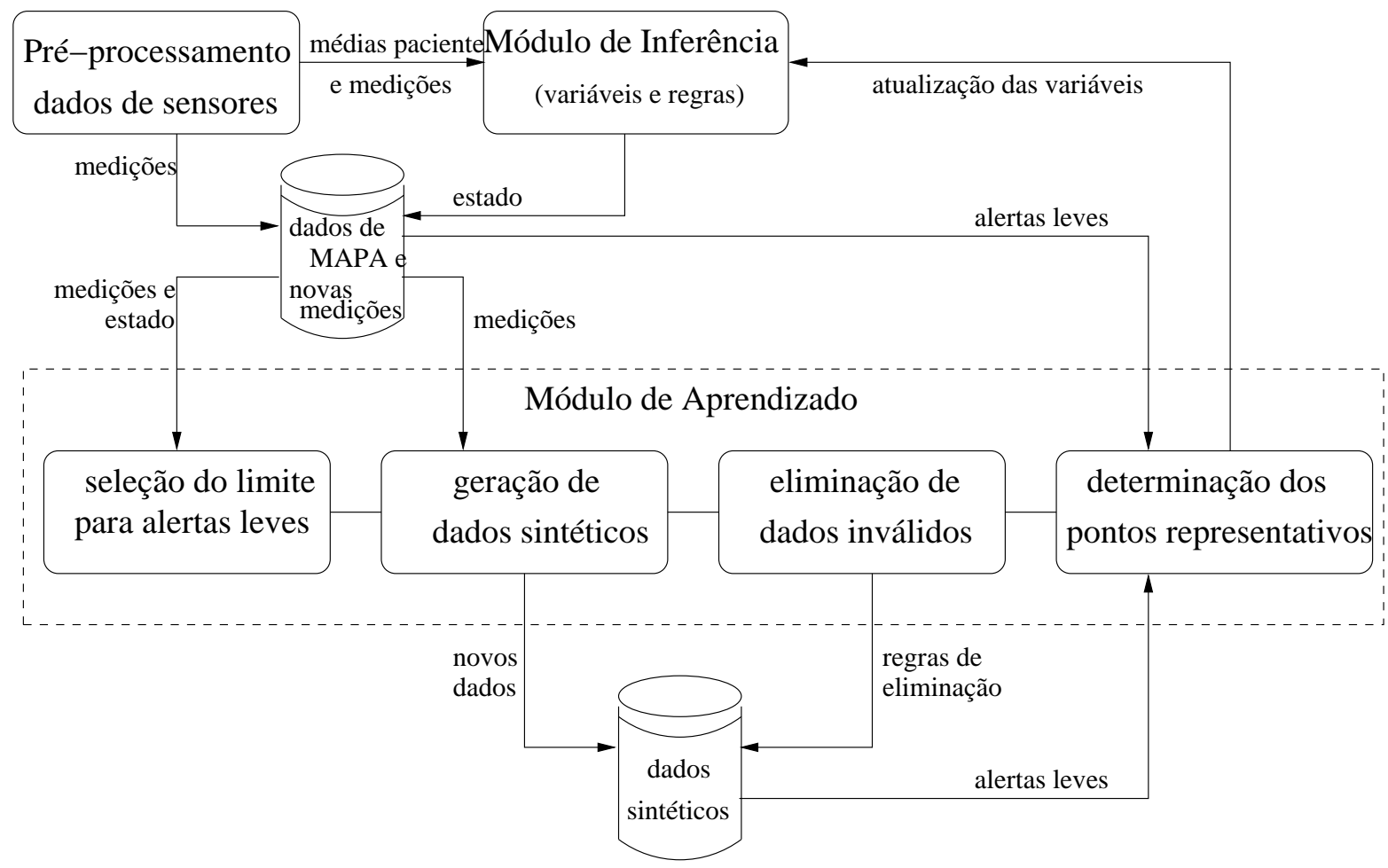

Figura 3: Fluxo para a tomada de decisão

Assim, no projeto do mecanismo de ajuste de regras foram definidas as três seguintes fases: 1) selecionar um subintervalo dos casos de alertas leves; 2) encontrar um ponto representativo; 3) ajustar as regras específicas às atividades. A seguir são discutidas cada uma dessas fases.

\subsubsection{Fase 1: Selecionar um subintervalo dos ca- sos de alertas leves}

A primeira decisão a ser tomada diz respeito a quais serão os dados usados pelo aprendizado. O médico atua nessa fase selecionando qual o subintervalo de casos de alerta estabeleceu-se como intervalo/faixa de alerta para o estado do paciente $(0,4 . .0,7)$. Por exemplo, o médico seleciona o subintervalo em que o estado seja $>0,4 \mathrm{e}<=0,65$. Isso satisfaz a seleção, pois o valor 0,4 é o valor obrigatório (valor original de limite da faixa para normalidade).

O recurso de seleção do subintervalo proporciona ao médico a possibilidade de expressar o grau de absorção dos casos de alerta pelo Modelo de Decisão. Para os testes feitos, adotouse o valor 0,65 como o limite para o subintervalo de casos de alerta (variável "maxAlertaLeve"). Com isso, quer-se que somente alertas leves sejam tratados pelo aprendizado. O valor 0,65 foi escolhido para contemplar casos que chegam a se aproximar dos casos de emergência. No entanto, somente os casos que mais ocorrem neste subintervalo contribuirão para determinar um ponto de ajuste.

\subsubsection{Fase 2: Encontrar um ponto representativo}

Nesta fase, depois de definida a variável "maxAlertaLeve", quer-se encontrar o ponto central (ou representativo) dos casos de alerta que se encontram nesse subintervalo. $\mathrm{O}$ ponto central é um conjunto de valores de variáveis usado como referência para ajustar as regras. Esse ajuste será feito no núcleo do conjunto "normal" da variável fuzzy. O ponto central deve atender as estratégias de projeto que dizem respeito a não utilização de casos extremos para o cálculo do ponto central e de propor ajustes sem cometer alterações em excesso.

No estudo de caso de monitoramento ora apresentado, independentemente da técnica escolhida para determinar o ponto central, os alertas ocorrem de duas formas: ou estão acima da média de pressão arterial ou estão abaixo. Nesses dois grupos tem-se que desconsiderar, ou dar menor importância, para valores extremos (outliers). Portanto, duas propriedades são desejáveis para a técnica: a eliminação de outliers e a definição de agrupamentos (clusters).

Outra questão diz respeito ao enfoque dado pelo trabalho para a PA, composta pela PAS e pela PAD. A PAS-PAD pos- 
sui uma singularidade entre outros sinais vitais, pois, no processamento da pressão arterial, os dois valores não estão dissociados. Por outro lado, também é possível tratar de forma isolada cada uma dessas variáveis.

Neste artigo, três diferentes técnicas serão testadas para encontrar o ponto representativo usando as variáveis do estudo de caso. O tratamento em separado da PAS e PAD será feito por uma técnica simples, denominada de média e desvio padrão (MDP). O tratamento em conjunto será feito por duas técnicas que usam agrupamentos: Fuzzy c-Means (FCM) e Gustafson-Kessel (GK). Essas duas técnicas foram escolhidas por diferirem no método para calcular a distância em relação ao ponto central. Com uma solução tomando as médias de PAS/PAD acima e/ou abaixo da média do paciente, obtemse valores em separado que podem não trazer a correlação PAS/PAD do paciente. Assim, uma questão a ser investigada é a eficiência de técnicas que tratam PAS e PAD de forma separada ou em conjunto.

\section{Média e desvio padrão (MDP)}

De acordo com estratégias de projeto, os valores mais distantes da média do conjunto de alertas não devem ser considerados no aprendizado. O método de média e desvio padrão faz esse controle por meio do desvio padrão.

No método MDP, de posse dos casos de alerta, separou-se os valores de PAS/PAD e, posteriormente, dividiu-se em valores acima e abaixo da média. São então formados quatro grupos, em torno da média geral de PAS/PAD do paciente. O primeiro grupo contém os valores de PAS superiores à média. No segundo grupo estão os valores de PAD superiores à média. O terceiro grupo contém os valores de PAS inferiores à média. No quarto grupo estão os valores de PAD inferiores à média. Os seguintes passos são executados para os grupos: 1) calcular a média; 2) calcular o desvio padrão; 3) eliminar valores extremos; 4) recalcular a média.

O procedimento do passo 3 é a eliminação dos pontos situados nas extremidades do agrupamento (outliers elimination). Para isso é utilizada a média acrescida (ou subtraída) de 1 desvio padrão. Assim, como regra, esse mecanismo utiliza $68 \%$ dos valores do conjunto de dados, assumindo uma distribuição normal.

Devido à simplicidade da MDP, essa técnica também pode ser usada para dados fisiológicos, como frequência cardíaca e peso. No entanto, devido ao relacionamento entre PAS e $\mathrm{PAD}$, é interessante explorar técnicas que proporcionem uma abordagem mais sofisticada de tratamento, o que é feito pelas técnicas de agrupamento.

\section{Agrupamento FCM}

Empregou-se dois algoritmos na determinação dos agrupamentos e, mais especificamente, para encontrar o ponto central do agrupamento: Fuzzy c-Means (FCM) (Bezdek, 1981), que usa a distância Euclidiana para o cálculo do ponto central; e Gustafson-Kessel (GK) (Gustafson e Kessel, 1978), que usa a distância de Mahalanobis para o cálculo do ponto central.

Na técnica de FCM, a função objetivo é minimizar a distância Euclidiana entre cada objeto de dados e seu centro do agrupamento. Um objetivo paralelo é maximizar a distância Euclidiana entre centros de agrupamentos (Ross, 1995). A distância Euclidiana entre os pontos $a$ e $b$, num espaço Euclidiano n-dimensional, tendo: $a=\left(x_{1}, x_{2}, \ldots, x_{n}\right) \mathrm{e}$ $b=\left(y_{1}, y_{2}, \ldots, y_{n}\right)$, é:

$$
d(a, b)=\sqrt{\left(x_{1}-y_{1}\right)^{2}+\left(x_{2}-y_{2}\right)^{2}+\cdots+\left(x_{n}-y_{n}\right)^{2}}
$$

Tanto na FCM quanto na GK é necessário escolher previamente qual quantidade de agrupamentos (c) que se quer formar. Definiu-se $c=2$, referindo-se ao agrupamento de valores acima da média e ao agrupamento de valores abaixo da média. Como já explicado, esse comportamento é esperado, devido ao fato de que os alertas só podem ser maiores ou menores do que a média do paciente.

A Figura 4 apresenta as possibilidades de concentração dos pontos de alerta. Dentro da área circular, que inclui a média geral do paciente, a possibilidade de casos de alertas é baixa. Externamente ao círculo, estão identificados os 4 quadrantes, usados para visualizar onde podem ocorrer agrupamentos de alertas. No entanto, assumiu-se que 2 pontos representativos (podendo estar em 2 quadrantes diferentes ou em 1 somente) são suficientes, pois quer-se obter um ponto que represente concentrações específicas de variações de pressão arterial. O que contribui para essa afirmação é a correlação entre PAS e PAD, investigada em Gavish et al. (2008). Com 2 pontos representativos é possível se ter um ponto acima e/ou abaixo da média, para o caso de PAS e/ou de PAD. Esse enquadramento também permite que se tenha uma PAS alta e uma PAD que se mantém inalterada - essa situação, conforme a experiência médica, geralmente ocorre em idosos. O ponto representativo, nesse caso, fica entre o $1^{\circ}$ e o $4^{\circ}$ quadrante.

Em um exemplo, tendo um paciente com média de PAS/PAD (em $\mathrm{mmHg}$ ) de $125 / 85$ e os resultados para os dois pontos representativos em 110/70 ( $3^{\circ}$ quadrante) e em 140/75 ( $4^{\circ}$ quadrante). Para a PAS, os dois pontos representativos dos alertas são 140 e 110: um valor acima e outro abaixo da média. Com isso, pode-se alterar o núcleo do conjunto normal da variável de PAS (em uma determinada atividade), tanto para cima quanto para baixo. Já no caso da PAD, o ajuste pode ser de 70 ou de 75 , pois ambos os valores são meno- 


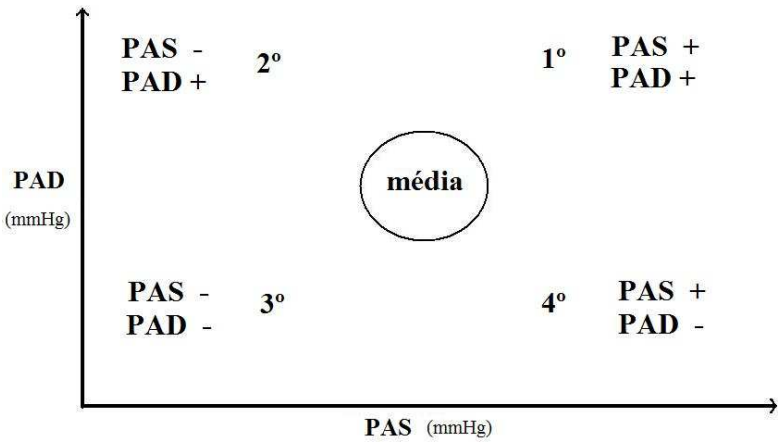

Figura 4: Áreas possíveis de agrupamentos de alertas

res do que a média de 85 . Portanto, a proposta de ajuste não apresenta ajuste para acima da média e somente um valor de PAD, como esperado, para abaixo da média. Para esse último, a escolha de um dos dois valores, sendo conservadora, usa como critério o valor mais próximo da média 85 , que é o 75. Assim, nos métodos de agrupamentos (incluindo o GK, que será mostrado), durante o cálculo do ponto representativo/central, os valores PAS/PAD são tratados em conjunto. No entanto, são separados no momento em que é feito o ajuste de cada variável.

\section{Agrupamento GK}

O algoritmo de Gustafson-Kessel também é uma técnica de agrupamento, que utiliza Mahalanobis na determinação do ponto central e é indicado para configurações de agrupamento aproximadamente hiperelipsoidais (Oliveira et al., 2007), enquanto o FCM é mais indicado para formas hiperesféricas. A distância de Mahalanobis distingue-se da distância Euclidiana por levar em conta as correlações do conjunto de dados. Essa característica pode ser interessante em relação à PAS-PAD, por determinar um centro do agrupamento com maior quantidade de pontos à sua volta. Outra vantagem é identificar um grupo que englobe dois quadrantes que estejam lado a lado, formando uma elipse.

A distância de Mahalanobis entre um grupo de valores com média $\mu=\left(\mu_{1}, \mu_{2}, \mu_{3}, \ldots, \mu_{p}\right)^{T}$ e matriz de covariância $\Sigma$ para um vetor multivariado $x=\left(x_{1}, x_{2}, x_{3}, \ldots, x_{p}\right)^{T}$ é definida como:

$$
D_{M}(x)=\sqrt{(x-\mu)^{T} \Sigma^{-1}(x-\mu)}
$$

\subsubsection{Fase 3: Ajustar as regras específicas às ati- vidades}

O ajuste de regras específicas para cada atividade é a última fase do aprendizado. Inicialmente, o ponto representativo (encontrado por qualquer uma das 3 técnicas apresentadas) é subtraído da média do paciente e resulta no desvio. Por exemplo, se o valor do ponto representativo de PAS é 140 e a média do paciente é 125 , então o desvio será $140-125=15$. O desvio será o parâmetro para a atualização de uma regra existente.

A seguir, é apresentado um exemplo de como as regras em atividades específicas podem ser ajustadas. Para tanto, tomou-se as regras de repouso em relação à PAS como exemplo: ${ }^{2}$

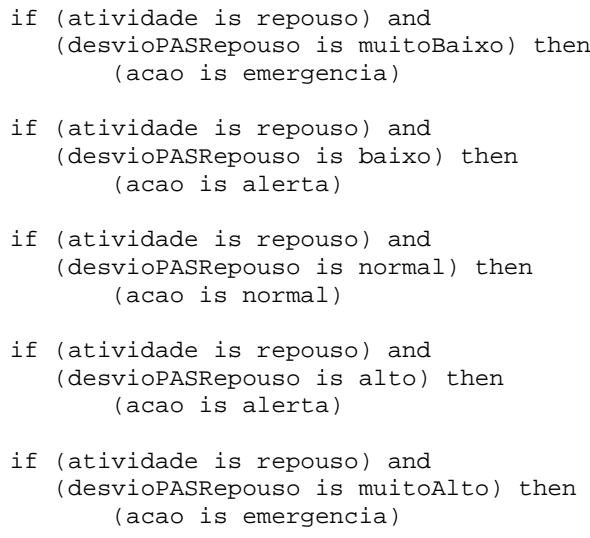

A variável desvioPASRepouso, que faz parte dessas regras, é mostrada na Figura 5-a e mais bem definida na Tabela 1.

Tabela 1: Variável desvioPASRepouso

\begin{tabular}{|c|c|c|}
\hline Termo & Função & Parâmetros \\
\hline muitoBaixo & Zmf $(z$-shaped $)$ & {$[-50 ;-20]$} \\
\hline baixo & Trapezoidal & {$[-50 ;-20 ;-10 ;-5]$} \\
\hline normal & Trapezoidal & {$[-10 ;-5 ; 5 ; 10,7]$} \\
\hline alto & Trapezoidal & {$[5 ; 10,7 ; 20 ; 50]$} \\
\hline muitoAlto & Smf $(s$-shaped $)$ & {$[20 ; 50]$} \\
\hline
\end{tabular}

A metodologia adotada para o ajuste é a de manter as distâncias entre os termos adjacentes estabelecidas nas regras iniciais, pois, assim, preserva-se o conhecimento médico incorporado ao modelo. Ao mesmo tempo, evita-se que ocorram sobreposições com total pertinência, como, por exemplo: $\exists x \mid \mu_{\text {normal }}(x)=\mu_{\text {alto }}(x)=1$, onde $\mu_{A}(x)$ é a pertinência de $x$ no conjunto $A$.

Para efetivar esta alteração de parâmetro do conjunto fuzzy, tem-se que observar alguns valores: a distância da borda direita do trapézio no termo "normal" é de 5, 7 (10,7 - 5), o

\footnotetext{
${ }^{2} \mathrm{O}$ ajuste é realizado somente nas variáveis fi siológicas de PAS e de PAD. Não foram descritas todas as variáveis, pois as cinco regras apresentadas nesse exemplo, envolvendo a variável "desvioPASrepouso", possuem a mesma confi guração para todas as demais variáveis.
} 

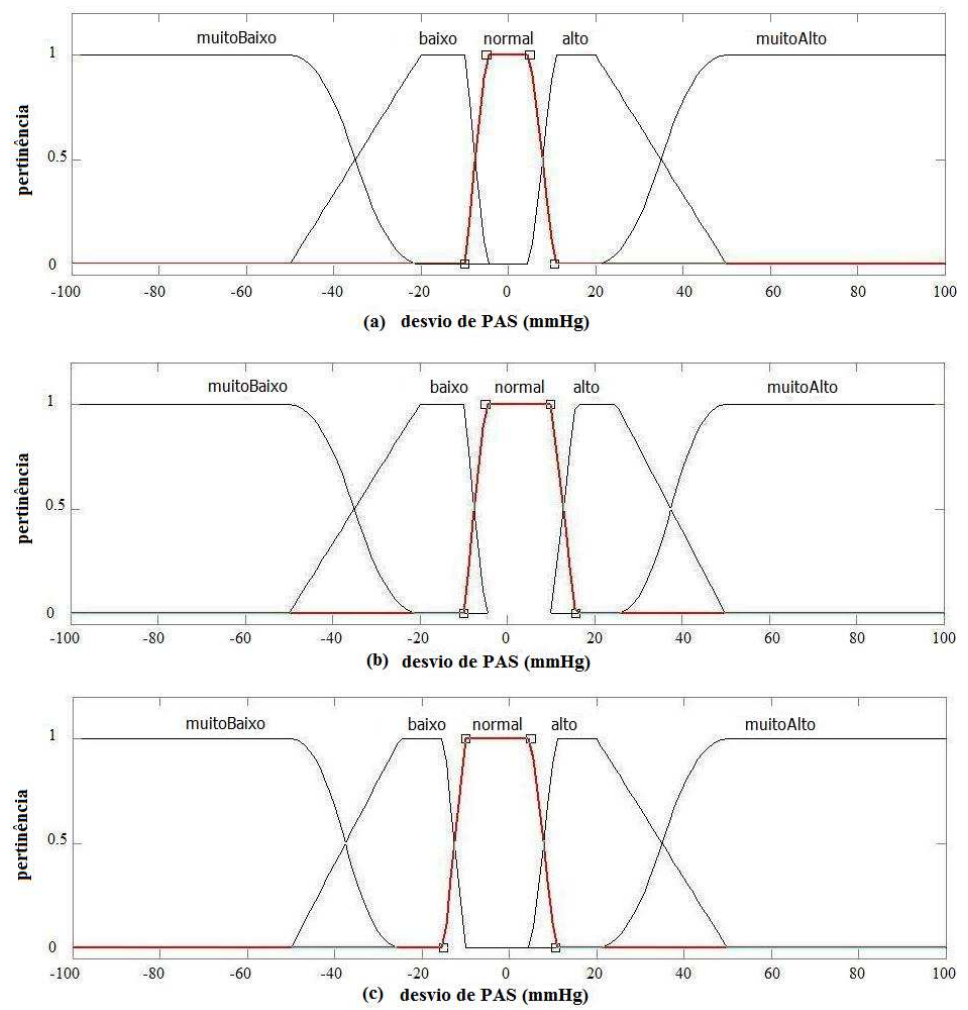

Figura 5: Variável de pressão arterial sistólica na atividade de repouso: (a) situação inicial, com o núcleo do conjunto normal em [-5,5]; (b) após o ajuste com desvio=10, o núcleo do "normal" passa a ser [-5,10]; (c) após o ajuste com desvio=-10, o núcleo do "normal" passa a ser [-10,5] 
que será denominado "bordaDireitaNormal"; e a "bordaEsquerdaNormal" é $5(|-10-(-5)|)$; além disso, a distância entre o terceiro e o segundo parâmetro do trapézio para o termo "alto" é $9,3(20-10,7)$, que corresponde ao núcleo do "alto". Com esses valores, pode-se aumentar o núcleo do "normal", ao mesmo tempo em que se mantém tanto a borda direita do termo "normal" quanto o núcleo do "alto". Um requisito final é não alterar a área de total pertinência do termo "muitoAlto", que implica uma emergência.

\section{AVALIAÇÃO DO MODELO}

Esta Seção apresenta a avaliação do mecanismo de Decisão por meio de testes com os Módulos de Inferência e de Aprendizado. Para viabilizar esses testes, é necessária a geração de dados sintéticos para a criação de vários cenários. Outra necessidade para o uso de dados sintéticos é que as técnicas usadas no Aprendizado precisam de uma grande quantidade de dados de entrada para produzirem resultados consistentes na saída. Dessa forma, pode-se realizar testes usando a simulação de vários dias de monitoramento, e ainda usar perturbações que simulem situações reais.

Os dados sintéticos são gerados com base nos dados reais de MAPA de 30 pacientes. O uso dos dados dos pacientes para essa pesquisa foi aprovado perante o Comitê de Ética em Pesquisa da Faculdade de Medicina/Hospital Universitário Antônio Pedro, da Universidade Federal Fluminense, por meio do parecer do CEP $n^{o} .103 / 09$. Para cada registro de uma MAPA, novos valores de PAS, PAD e FC são gerados, mas o padrão da variável atividade é mantido. Essa estratégia está em sintonia com a solução proposta, pois associou-se os dados fisiológicos ao comportamento. Ao contrário, caso uma nova atividade seja gerada, perde-se a associação entre essas classes de variáveis.

A fim de dar suporte à análise dos dados, a Figura 6 apresenta as médias de pressão arterial e a sequência de pacientes ordenada crescentemente pela média geral de $P A S+P A D$. Os 4 primeiros pacientes possuem valores baixos de pressão arterial. Efetivamente, eles não são considerados hipotensos, pois as informações de pressão não estão associadas a um relato de sintoma. Assim, considerou-se do $1^{\circ}$ ao $15^{\circ}$ paciente casos de normotensos e do $16^{\circ}$ ao $30^{\circ}$ casos de hipertensos.

\subsection{Avaliação do Módulo de Inferência}

A Tabela 2 apresenta os resultados do sistema de identificação de alarmes para os dados de MAPA pelo método tradicional e pelo sistema proposto neste artigo (Módulo de Inferência). Os pacientes foram classificados considerando as diretrizes apresentadas em SBC (2010), que estabelecem como hipertensos no monitoramento residencial os pacientes

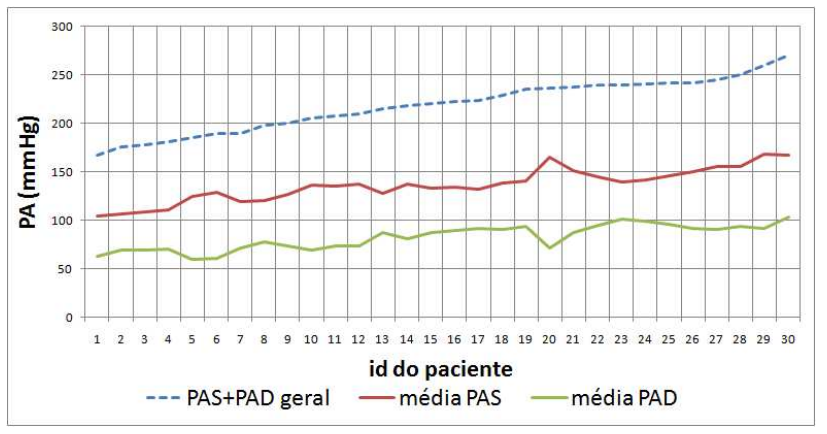

Figura 6: Médias de PA com os pacientes ordenados pela soma PAS e PAD

com pressão arterial $>135 / 85 \mathrm{mmHg}$. No método tradicional, todas as medições acima de $135 / 85 \mathrm{mmHg}$ na vigília e $120 / 70 \mathrm{mmHg}$ no sono são consideradas alertas. As emergências não são identificadas e fica a cargo do médico fazer essa análise posteriormente. Adotou-se aqui o termo "método tradicional", porque se trata da forma atual de geração de relatórios por sistemas que processam os dados gerados pela MAPA.

Definiu-se o percentual de alarmes como sendo:

$\%$ alarmes $=(($ alertas + emergencias $) /$ normais $) * 100$

$\mathrm{O}$ sistema acusou um percentual de alarmes maior do que o da análise tradicional de MAPA somente nos 4 primeiros pacientes, os quais possuem valores baixos de pressão. Isso ocorre porque o método tradicional de relatórios de MAPA não identifica os valores baixos de pressão. Já a alta quantidade de alarmes no método tradicional ocorre porque este não considera a atividade sendo praticada nem a média do paciente, entre outros fatores.

Outra contribuição do Módulo de Inferência é a identificação de emergências que determinariam novas medições e talvez a notificação de profissionais de saúde. Portanto, o conceito de emergência é adicional à análise tradicional de MAPA e reforça o mecanismo de identificação de situações críticas. De forma complementar, esse recurso pode ser acompanhado de um botão de pânico que é acionado pelo paciente, para reforçar a ocorrência da situação crítica.

Outros testes foram feitos, criando alguns cenários de variações de sinais vitais e gerando dados sintéticos com a distribuição uniforme. Com isso, conseguiu-se testar limites para as variáveis. Por questão de espaço, esses testes não são mostrados neste trabalho. Os resultados apresentados pelo Módulo de Inferência demonstram que o sistema possui características interessantes para o monitoramento de longa duração. A identificação de alarmes se concentra nas grandes variações, pois, além de usar a média do paciente, respeita uma margem de tolerância que depende da atividade sendo praticada minutos antes da medição. Dessa forma, os pacientes 


\begin{tabular}{|c|c|c|c|c|c|c|c|c|}
\hline & \multicolumn{3}{|c|}{ Tradicional } & \multicolumn{3}{|c|}{ Sistema } & \multicolumn{2}{|c|}{ \%alarmes } \\
\hline id & normais & alertas & emerg. & normais & alertas & emerg. & Tradic. & Sistema \\
\hline 1 & 76 & 3 & - & 59 & 19 & 1 & 4 & 34 \\
\hline 2 & 70 & 11 & - & 54 & 25 & 2 & 16 & 50 \\
\hline 3 & 48 & 10 & - & 41 & 16 & 1 & 21 & 41 \\
\hline 4 & 53 & 3 & - & 45 & 9 & 2 & 6 & 24 \\
\hline 5 & 53 & 22 & - & 57 & 16 & 2 & 42 & 32 \\
\hline 6 & 66 & 12 & - & 66 & 11 & 1 & 18 & 18 \\
\hline 7 & 50 & 29 & - & 58 & 20 & 1 & 58 & 36 \\
\hline 8 & 39 & 26 & - & 49 & 13 & 3 & 67 & 33 \\
\hline 9 & 50 & 21 & - & 56 & 8 & 7 & 42 & 27 \\
\hline 10 & 16 & 65 & - & 67 & 14 & 0 & 406 & 21 \\
\hline 11 & 33 & 45 & - & 50 & 22 & 6 & 136 & 56 \\
\hline 12 & 20 & 41 & - & 41 & 18 & 2 & 205 & 49 \\
\hline 13 & 19 & 38 & - & 37 & 15 & 5 & 200 & 54 \\
\hline 14 & 15 & 42 & - & 34 & 23 & 0 & 280 & 68 \\
\hline 15 & 8 & 56 & - & 46 & 18 & 0 & 700 & 39 \\
\hline
\end{tabular}

\begin{tabular}{|c|c|c|c|c|c|c|c|c|}
\hline \multirow[b]{2}{*}{ id } & \multicolumn{3}{|c|}{ MAPA } & \multicolumn{3}{|c|}{ Sistema } & \multicolumn{2}{|c|}{ \%alarmes } \\
\hline & normais & alertas & emerg. & normais & alertas & emerg. & Tradic. & Sistema \\
\hline 16 & 6 & 69 & - & 58 & 14 & 3 & 1150 & 29 \\
\hline 17 & 1 & 79 & - & 62 & 17 & 1 & 7900 & 29 \\
\hline 18 & 7 & 54 & - & 37 & 22 & 2 & 771 & 65 \\
\hline 19 & 6 & 75 & - & 45 & 36 & 0 & 1250 & 80 \\
\hline 20 & 1 & 68 & - & 39 & 24 & 6 & 6800 & 77 \\
\hline 21 & 8 & 71 & - & 35 & 41 & 3 & 888 & 126 \\
\hline 22 & 2 & 77 & - & 53 & 26 & 0 & 3850 & 49 \\
\hline 23 & 2 & 55 & - & 38 & 16 & 3 & 2750 & 50 \\
\hline 24 & 8 & 50 & - & 25 & 32 & 1 & 625 & 132 \\
\hline 25 & 3 & 77 & - & 58 & 22 & 0 & 2567 & 38 \\
\hline 26 & 1 & 59 & - & 37 & 18 & 5 & 5900 & 62 \\
\hline 27 & 1 & 57 & - & 32 & 25 & 1 & 5700 & 81 \\
\hline 28 & 4 & 75 & - & 29 & 48 & 2 & 1875 & 172 \\
\hline 29 & 1 & 45 & - & 20 & 26 & 0 & 4500 & 130 \\
\hline 30 & 0 & 63 & - & 25 & 38 & 0 & - & 152 \\
\hline
\end{tabular}

Tabela 2: Avaliação dos dados de MAPA pelo método tradicional e pelo sistema

hipertensos são tratados de forma diferenciada, permitindo que os médicos se concentrem nos casos que demandem uma análise das causas das variações de pressão arterial.

É oportuno ainda um comentário geral sobre as emergências de alguns testes realizados com o Módulo de Inferência. O número elevado de emergências, mesmo com poucas variações, ocorre em pacientes com picos de pressão e, nesse caso, trata-se de pacientes com pressão arterial média considerada normal. Quando o paciente não identifica que a atividade nos minutos precedentes envolveu um esforço físico, essa falta de informação contribui para que a situação seja identificada como um alerta ou emergência. Além de picos naturais de pressão, que incluem, principalmente, as situações de estresse, as quais não são tratadas por este trabalho, esses picos podem ser também causados pelo efeito do avental branco ou pelos erros no momento da medição.

\subsection{Avaliação do Módulo de Aprendizado}

O Módulo de Aprendizado, descrito por meio das fases apresentadas na Subseção 5.2, inicia após a seleção do subintervalo dos alertas, que define o valor mais alto do estado do paciente para ser considerado um alerta leve (variável maxAlertaLeve). Foi usado: maxAlertaLeve $=0,65$. A segunda fase é encontrar um ponto representativo. Para isso, gerou-se dados sintéticos conforme apresentado na Subseção 5.1. Esses dados são agregados aos dados de medições, que envolve tanto dados de MAPA quanto medições realizadas durante o funcionamento do monitoramento. Em seguida, uma das três técnicas, que são testadas nesta Subseção, encontrará pontos representativos para as variáveis PAS e PAD. As técnicas separam o conjunto de dados por atividade e trabalham somente com os casos em que o estado do paciente é $(0,4 . .0,65]$.
Os testes com o Módulo de Aprendizado têm por objetivo validar o mecanismo de ajuste de regras. Como resultado, quer-se definir uma melhor técnica entre as estudadas: MDP, FCM ou GK. A implementação da técnica MDP foi realizada usando código em C\#; a implementação da técnica FCM foi feita no Matlab; já o algoritmo de GK foi implementado adaptando o código apresentado em Abonyi e Feil (2007).

A Figura 7 apresenta os resultados para as duas técnicas de agrupamento para um paciente na atividade de repouso. Os círculos identificam a posição do ponto central/representativo para o FCM, e o asterisco, para o GK. A técnica de agrupamento GK não consegue definir um valor baixo para a PAS, pois o valor 132 obtido está acima da média 127. Observou-se que o GK não atribui grande relevância para o conjunto disperso com baixos valores PAS e PAD $\left(3^{\circ}\right.$ quadrante). Assim, o GK não apresenta um ponto representativo para um ajuste abaixo da média. Apesar de não propor um dos ajustes, isso pode ser entendido como um fator de estabilidade, pois não serão propostos ajustes com base em dados pouco aglomerados.

\subsection{Avaliação médica da proposta}

Para uma avaliação final do mecanismo de Decisão, dois médicos realizaram uma classificação dos dados originais de MAPA dos 30 pacientes. Os médicos (aqui denominados médico A e médico B) classificaram cada medida em normal, alerta ou emergência. Cabe ressaltar que o médico B teve o acompanhamento de mais um terceiro médico, mas a avaliação foi reunida em uma só. As avaliações médicas pressupuseram que as medidas de MAPA foram obtidas em momentos diferentes do dia. Para efeito de análise, isso quer dizer que a coleta de cada medida não obedece a um intervalo de tempo fixo entre as medidas. 


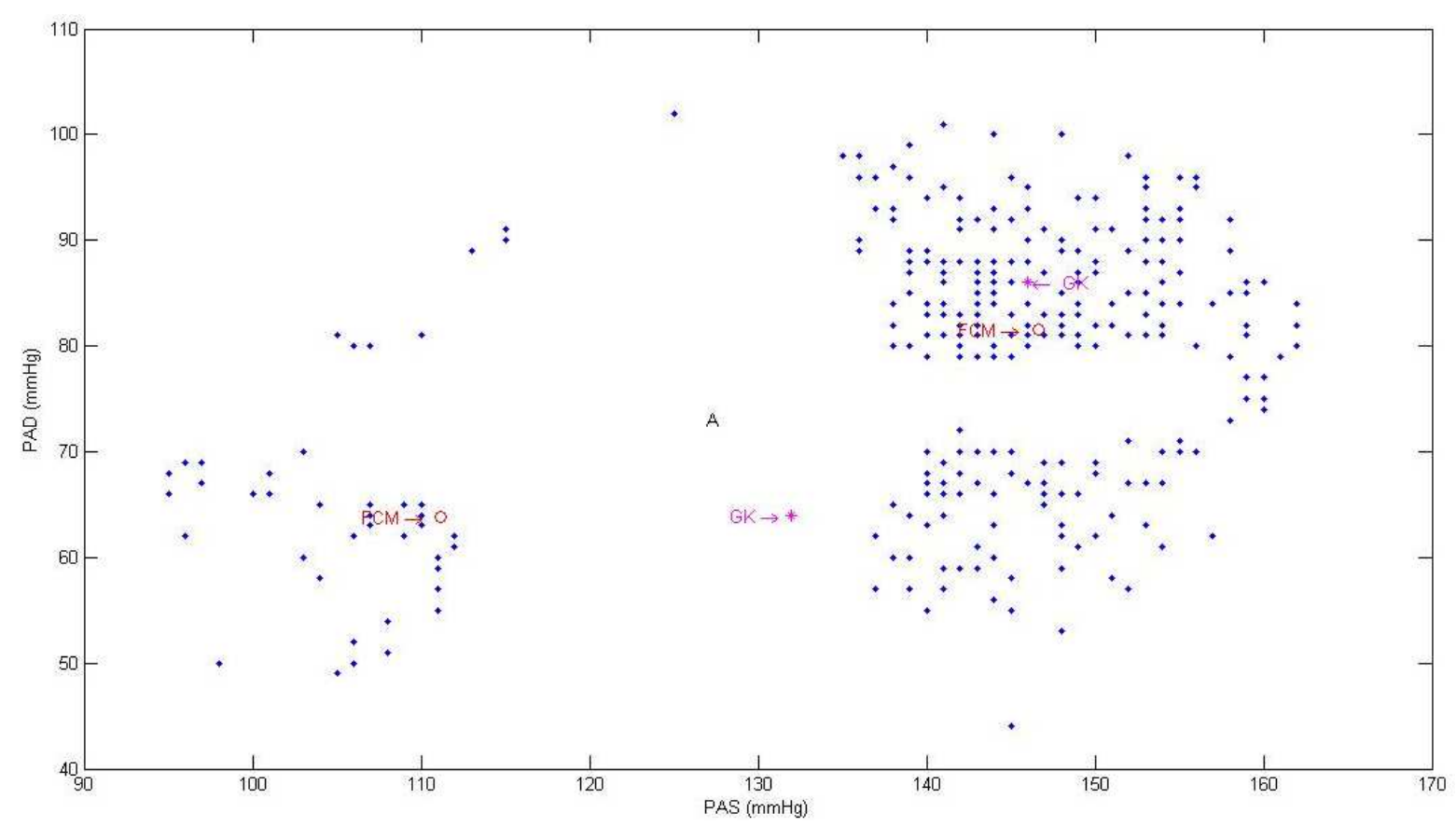

Figura 7: Pontos centrais para o paciente com id 9, em repouso. A média do paciente é 127/73 ('A') e os resultados obtidos com o FCM foram: 147/82 e 112/64, para o GK foram: 146/86 e 132/64, e para o MDP foram: 143/83 e 107/64

Após a avaliação médica, o sistema realizou execuções nas seguintes situações: a) antes do uso do aprendizado; b) após o uso do aprendizado. A execução antes do uso do aprendizado compreende o conjunto de regras iniciais. Nessa situação, espera-se uma maior intolerância às variações de dados fisiológicos do paciente. Após o aprendizado, serão obtidas as respostas das 3 técnicas propostas e uma expectativa de tolerância maior às variações.

O resultado esperado para o sistema é a identificação correta de casos normais (classe 'N'), alertas (classe 'A') e emergências (classe 'E'). Um fator crítico é o médico identificar uma emergência e o sistema identificar como normal, o que caracteriza um falso negativo. Por sua vez, o sistema identificar uma emergência não detetada pelo médico tende a ser menos prejudicial, mas caracteriza um falso positivo. Nesse caso, o sistema poderia solicitar uma nova medição para confirmar o resultado e só então tomar uma decisão quanto à emergência.

As Tabelas 3, 4, 5 e 6 apresentam as matrizes de confusão contendo as avaliações médicas (nas linhas) e os resultados do sistema (nas colunas). Por simplificação, nas tabelas "após o aprendizado", foram colocados os resultados do sistema somente para a técnica de FCM, já que os resultados obtidos são parecidos com as técnicas GK e MDP e podem ser explicados posteriormente. Para melhor entendimento, na Tabela 3 pode-se ver que o médico A identificou 10 casos de emergência e o sistema classificou 1 deles como alerta. Já o médico B (Tabelas 5 e 6) não identificou casos de emergência.

Tabela 3: Matriz de Confusão - médico A, antes do aprendizado

\begin{tabular}{|c|c|c|c|}
\hline Avaliação & \multicolumn{3}{|c|}{ Sistema } \\
\hline Médica & N & A & E \\
\hline N & 1267 & 530 & 6 \\
\hline A & 1 & 58 & 30 \\
\hline E & 0 & 1 & 9 \\
\hline
\end{tabular}

Tabela 4: Matriz de Confusão - médico A, após o aprendizado com FCM

\begin{tabular}{|c|c|c|c|}
\hline Avaliação & \multicolumn{3}{|c|}{ Sistema } \\
\hline Médica & N & A & E \\
\hline N & 1648 & 149 & 6 \\
\hline A & 2 & 52 & 35 \\
\hline E & 0 & 0 & 10 \\
\hline
\end{tabular}

Em todos os cenários, não estão presentes casos de falso negativo (emergências em que o sistema classificou como normais). Esse é um ponto de extrema importância para o sistema, pois é um dos fatores para a sua confiabilidade.

A acurácia foi usada para comparar os resultados antes e após o aprendizado, sendo definida como a soma dos casos corretamente classificados (isto é, a soma da diagonal principal 
Tabela 5: Matriz de Confusão - médico B, antes do aprendizado

\begin{tabular}{|c|c|c|c|}
\hline Avaliação & \multicolumn{3}{|c|}{ Sistema } \\
\hline Médica & N & A & E \\
\hline N & 1268 & 560 & 17 \\
\hline A & 0 & 29 & 28 \\
\hline E & 0 & 0 & 0 \\
\hline
\end{tabular}

Tabela 6: Matriz de Confusão - médico B, após o aprendizado com FCM

\begin{tabular}{|c|c|c|c|}
\hline Avaliação & \multicolumn{3}{|c|}{ Sistema } \\
\hline Médica & N & A & E \\
\hline N & 1647 & 175 & 23 \\
\hline A & 3 & 26 & 28 \\
\hline E & 0 & 0 & 0 \\
\hline
\end{tabular}

da matriz de confusão) dividida pelo número total de casos. $\mathrm{Na}$ situação antes do aprendizado, a acurácia do sistema é de $70 \%$ para o médico A e $68 \%$ para o médico B.

Após aplicar o aprendizado, o resultado alcançado no médico A foi de $90 \%$ de acurácia na técnica FCM, $88 \%$ na GK e $86 \%$ na MDP. Os resultados para o médico B são, exatamente, 2 pontos percentuais a menos do que os valores apresentados para o médico A.

Um último teste foi realizado, selecionando somente os casos que houve consenso entre os médicos na avaliação. Nesse caso, a acurácia foi de $91 \%$.

O número de emergências após o aprendizado se manteve igual ao número de casos antes do aprendizado. Assim, mesmo com o ajuste nos casos de alertas, o sistema cumpriu com sua proposta de manter a rigidez para os casos de emergências e, ao mesmo tempo, tolerar variações consideradas leves para o paciente do ponto de vista médico.

Após o aprendizado, pode-se ver que existem ainda 2 casos (Tabela 4) e 3 casos (Tabela 6) considerados como alertas pelos médicos e classificados como normais pelo sistema. Desses casos, existe somente 1 caso em que os dois médicos concordaram que se trata de um alerta. Esse caso é de uma PAD baixa em que o paciente informou que estava dormindo. Devido à atividade, o sistema classificou a situação no limite entre normal e alerta. Como é a primeira medida que inicia o período de sono informado pelo paciente, pode ser que ainda ele não estivesse dormindo, o que resultaria um alerta pelo sistema. Os outros casos classificados como normais também se enquadram nessa possibilidade de uma entrada informada de forma incorreta pelo paciente.
A técnica de FCM obteve os melhores resultados, pois o número de casos detectados como alertas (e considerados normais pelos médicos) é menor do que a GK e a MDP. No ajuste do núcleo dos conjuntos normais em cada variável ajustada, a FCM faz um ajuste maior do que o realizado pelas demais técnicas. A razão para isso é que a FCM consegue um maior distanciamento entre os pontos propostos de ajuste, ao mesmo tempo em que acompanha a correlação entre PAS e PAD. O resultado intermediário da técnica GK demonstra que o acompanhamento da correlação também é mais efetivo do que simplesmente tomar a média dos pontos, como faz a MDP. A FCM, usando a distância Euclidiana no cálculo do ponto representativo, consegue posicionar, na maior parte das situações, dois pontos em dois quadrantes. Já na GK, usando a distância de Mahalanobis, quando há uma grande concentração de pontos em um quadrante, os pontos representativos tendem a ficar no mesmo quadrante. Com isso, a FCM propõe um valor acima e abaixo da média, o que possibilita abranger mais casos de alertas para tornarem-se normais.

\section{CONCLUSÕES}

Este trabalho apresentou um mecanismo inédito de decisão para inferência de contexto em aplicações de monitoramento de saúde em ambientes pervasivos. O monitoramento proposto contempla regras que realizam a fusão de dados ambientais, fisiológicos e comportamentais. Dessa forma, o sistema proporciona uma importante ferramenta para o apoio à análise médica, já que diversas variáveis podem ser contempladas na solução, devido a flexibilidade do sistema. A identificação de situações críticas é realizada em tempo real e contínua, sendo supervisionada pelo médico, o qual controla em alto nível (por meio do conceito de alertas leves) o desempenho do mecanismo de Decisão.

Um grande desafio em sistemas de monitoramento de saúde que utilizam regras é realizar o aprendizado sem prejudicar a interpretabilidade das regras. A proposta apresentada resolve esse problema com um conjunto de medidas: o sistema de inferência usa poucas variáveis no antecedente de cada regra, os ajustes são realizados sob supervisão médica e, por último, o aprendizado ocorre com base em pontos representativos que servem para ajustar regras específicas. A estratégia de tornar normais os casos de alertas leves permite que o sistema seja utilizado no monitoramento individualizado de longa duração.

O sistema apresentado deve ser testado com muitos pacientes, em situações reais de monitoramento em casa. Notouse que inexistem bases de dados contendo o monitoramento diário de uma pessoa por um longo tempo. Para o aprendizado, tentou-se diminuir essa falta de dados utilizando dados sintéticos que reproduzem o comportamento dos dados reais 
disponíveis em cada atividade. Isso pode reduzir o período de adaptação do sistema para o indivíduo sendo monitorado, mas mais testes são necessários. Seguindo nesse caminho, é pretendido organizar um banco de dados de medidas e disponibilizar o conjunto de regras para o monitoramento da pressão arterial. Com isso, outros grupos de pesquisa podem propor mudanças, incluindo novas variáveis, como outros sinais vitais e atividades diárias do paciente, ou mesmo o controle de outras doenças crônicas.

\section{AGRADECIMENTOS}

Os autores gostariam de agradecer o apoio financeiro recebido da FAPERJ e CNPq, bem como aos colaboradores médicos que proveram o conhecimento especializado essencial para a realização dessa pesquisa.

\section{REFERÊNCIAS}

Abonyi, J. e Feil, B. (2007). Cluster Analysis for Data Mining and System Identification, Birkhäuser, Basileia, Suíça.

Anagnostopoulos, C. e Hadjiefthymiades, S. (2010). Advanced fuzzy inference engines in situation aware computing, Fuzzy Sets Systems 161(4): 498-521.

Bezdek, J. C. (1981). Pattern Recognition with Fuzzy Objective Function Algorithm, Plenum Press, Nova York, EUA.

Copetti, A., Leite, J. C. B., Loques, O. G., da Nóbrega, A. C. L. e de P. C. Barbosa, T. (2009). Intelligent context-aware monitoring of hypertensive patients, Situation Recognition and Medical Data Analysis in Pervasive Health Environments. Pervasive Healthcare Conference, IEEE, Londres, Reino Unido, pp. 1-6.

da Nobrega, A. C. L. (2005). The subacute effects of exercise: concept, characteristics, and clinical implications, Exercise and Sport Sciences Reviews 33(2): 84-87.

Dey, A. (2001). Understanding and using context, Personal and Ubiquitous Computing 5(1): 4-7.

Duchêne, F., , Garbay, C. e Rialle, V. (2007). Learning recurrent behaviors from heterogeneous multivariate timeseries, Artificial Intelligence in Medicine 39(1): 25-47.

El-Samahy, E., Mahfouf, M. e Linkens, D. A. (2006). A closed-loop hybrid physiological model relating to subjects under physical stress, Artificial Intelligence in Medicine 38(3): 257-274.

Figueiredo, K., Vellasco, M., Pacheco, M. e Souza, F. (2007). Modelo neuro-fuzzy hierárquico politree com aprendi- zado por reforço para agentes inteligentes, Revista Controle \& Automação, Sociedade Brasileira de Automática 18: 234-250.

Gavish, B., Ben-Dov, I. Z. e Bursztyn, M. (2008). Linear relationship between systolic and diastolic blood pressure monitored over $24 \mathrm{~h}$ : assessment and correlates, Journal of Hypertension 26(2): 199.

Gustafson, D. E. e Kessel, W. C. (1978). Fuzzy clustering with a fuzzy covariance matrix, IEEE Conference on Decision and Control, pp. 761-766.

Hoffmann, F. (2001). Evolutionary algorithms for fuzzy control system design, Proceedings of the IEEE 89(9): 1318-1333.

IBGE (2009). Instituto Brasileiro de Geografia e Estatística - Indicadores Sociodemográficos e de Saúde no Brasil. http: //www.ibge.gov.br.

Lee, H., Park, K., Lee, B., Choi, J. e Elmasri, R. (2008). Issues in data fusion for healthcare monitoring, 1st International Conference on Pervasive Technologies Related to Assistive Environments, Atenas, Grécia, pp. 3:1-3:8.

Leijdekkers, P., Gay, V. e Lawrence, E. (2007). Smart homecare system for health tele-monitoring, 1st International Conference on the Digital Society, Guadalupe, França.

Marschollek, M., Ludwig, W., Schapiewksi, I., Schriever, E., Schubert, R., Dybowski, H., zu Schwabedissen, H. M., Howe, J. e Haux, R. (2007). Multimodal home monitoring of elderly people-first results from the LASS study, IEEE 21st International Conference on Advanced Information Networking and Applications, Workshop on Smart Homes for Tele-Health, Niagara Falls, Canadá, pp. 815-819.

Mion, D., Oigman, W. e Nobre, F. (2004). MAPA: Monitorização Ambulatorial da Pressão Arterial, Atheneu, Rio de Janeiro.

Norris, P. R. (2006). Toward New Vital Signs: Tools and Methods for Physiologic Data Capture, Analysis, and Decision Support in Critical Care, PhD thesis, Biomedical Informatics, Vanderbilt University, Nashville, EUA.

Oliveira, H. A. E., Machado, M. A. S., Caldeira, A. M., Souza, R. C. e Tanscheit, R. (2007). Inteligência Computacional Aplicada à Administração, Economia e Engenharia em Matlab, Thomson Learning, São Paulo.

ONU (2009). Population Division of the Department of Economic and Social Affairs of the United Nations Secretariat - World Population Prospects: The 2008 Revision. http://esa.un.org/unpp/. 
Pentney, W., Popescu, A. M., Wang, S., Kautz, H. e Philipose, M. (2006). Sensor-based understanding of daily life via large-scale use of common sense, 21st National Conference on Artificial Intelligence, Boston, EUA, pp. 906-912.

Pärkkä, J., Merilahti, J., Mattila, E. M., Malm, E., Antila, K., Tuomisto, M. T., Saarinen, A. V., van Gils, M. e Korhonen, I. (2009). Relationship of psychological and physiological variables in long-term self-monitored data during work ability rehabilitation program, Information Technology in Biomedicine 13(2): 141-151.

Ross, T. (1995). Fuzzy logic with engineering applications, McGraw-Hill, Nova York, EUA.

Ruscio, J. e Haslam, N. (2006). Introduction to the Taxometric Method: A Practical Guide, Lawrence Erlbaum, Mahwah, EUA.

SBC (2005). Sociedade Brasileira de Cardiologia. IV Diretriz para uso da Monitorização Ambulatorial da Pressão Arterial. II Diretriz para uso da Monitorização Residencial da Pressão Arterial, Arquivos Brasileiros de Cardiolologia 85(Supl II): 1-18.

SBC (2010). VI Diretrizes Brasileiras de Hipertensão, Arquivos Brasileiros de Cardiologia 95(1 supl. 1): 1-51.

Sneha, S. e Varshney, U. (2009). Enabling ubiquitous patient monitoring: model, decision protocols, opportunities and challenges, Decision Support Systems 46(3): 606619.

Sztajnberg, A., Rodrigues, A. L. B., Bezerra, L. N., Loques, O. G., Copetti, A. e Carvalho, S. T. (2009). Applying context-aware techniques to design remote assisted living applications, International Journal of Functional Informatics and Personalised Medicine 2(4): 358-378.

Tsipouras, M. G., Voglis, C. e Fotiadis, D. I. (2007). A framework for fuzzy expert system creation-application to cardiovascular diseases, IEEE Transactions on Biomedical Enginnering 54(11): 2089. 